\title{
Partition and Efficiency Sample in Statistical Models by Entropy
}

\author{
Mohammed Elmoudier Mohammed Gobar \\ Mathematics Department, Faculty of Science and Arts, Al-Baha University, KSA.
}

\begin{abstract}
In this paper we show that how the determination of the $\delta$-sufficient partition of sample space when the information is measured by entropy. Also, the efficiency sample for a given random variable in discrete form in a given probability space can be done for different distributions. Testing example for the given method is considered in case the random sample have different distributions.
\end{abstract}

Keywords: Random sample; entropy measures; efficiency of statistical models.

2010 AMS subject classifications: 94A17,94A20.

\section{INTRODUCTION}

In treating statistical models, the summarizing of the given data by means of statistical methods such as frequency table, histogram...etc, many information in the model will be lost,this amount of loss should be very small in order to get more correct results. The measurement of the amount of loss information is of the most important concepts in such fields. This paper aims to derive some results which are similar to those in [2],[6] when the amount of information in the data is measured by the entropy measures defined by Vavrda and Charval [8], Forte [5] and Behara and Chawla [4]. Moreover, the suggested method in [6] is used to evaluate the efficiency of a random sample and to determine the sample size in order to obtain a sample of a given efficiency. The assumed procedure is tested in the case of geometric and exponential distributions. 
Let $\mathrm{X}$ be a random variable defined on a given probability space $(\Omega, \mathcal{F}, P)$ with probability density function (p.d.f) $f(x)$. Assume that the space of $X$ is $S$. Let $f$ be $\sigma$-field in $\mathrm{S}$ and $\alpha$ be a positive real number different from one, then the amount of uncertainty in the information represented by $\mathrm{X}$ can be measured by the following three entropy measures:

(1) Vavrda and Charval (1967) measure

$$
H_{1}(x)=E(f(x))^{\alpha-1}
$$

(2) The Forte (1969) average certainty

$$
H_{2}(x)=2^{\alpha E(\log (f(x)))}
$$

(3) Behara and Chawla (1974) entropy measure

$$
H_{3}(x)=\frac{1}{1-\alpha}\left(\sum_{\alpha \neq 1} f(x)(f(x))^{(\alpha-1)}-1\right)
$$

The partitioning of the sample space of the model consideration plays an essential role. The comparison between assumed entropy measures of this partition and that of the corresponding model is proved in Theorem (3.1).

\section{DEFINITIONS AND NOTATIONS}

In this section, we present the needed definitions and results for the coming sections.

Definition 1. Let $X$ be a random variable in a given probability space $(\Omega, \mathcal{F}, P)$ with (p.d.f) $f(X ; \lambda)$. Let $\rho=\left\{S_{1}, \ldots, S_{n}\right\}$ such that $S_{j} \in \mathcal{F}$ for all $j=1, \ldots, n$ with $S_{i} \cap S_{j}=\emptyset$ for all $i \neq j$ and $\cup_{j=1}^{n} S_{j}=S$ and $\mathcal{F}$ is $\sigma-$ field of events in $S$. For each $j=1, \ldots, n$,then $\rho$ is called an $\mathcal{F}$-partition of $S$.

Definition 2. Let $\rho$ be an $\mathcal{F}$-partition for the space of a random variable $X, \rho$ is called a $\delta$-sufficient partition with respect to the some information measure $H(\lambda)$ iff for some real number $\delta$ such that $0<\delta<1$,

$$
\frac{H(\lambda, \rho)}{H(\lambda)}=\delta
$$

If $\delta=1$ then $\rho$ is called a sufficient partition with respected to information measure $H(\lambda)$. Note that $(1-\delta)$ represents the relative loss of information due to the partition $\rho$. Moreover, $\delta$ may be called the efficiency of the partition $\rho$ with respect to the entropy [2]. 
Definition 3. Let $\rho=\left\{S_{1}, \ldots, S_{n}\right\}$ be an $\mathcal{F}$-partition of the space of a random variable X with p.d.f. $f(X ; \lambda)$ Set

$$
\mu\left(S_{j} ; \lambda\right)=P\left(X \in S_{j}\right) \text { for each } j=1, \ldots, n .
$$

and

$$
g_{j}(x ; \lambda)= \begin{cases}\frac{f(x ; \lambda)}{\mu\left(S_{j} ; \lambda\right)} & x \in S_{j} \\ 0 & \text { otherwise. }\end{cases}
$$

Note that $g_{j}(x ; \lambda)$ is a p.d.f. on $S_{j}$ for each $j=1, \ldots, n$ and $\left\{\mu\left(S_{1} ; \lambda\right), \ldots, \mu\left(S_{n} ; \lambda\right)\right\}$ is a discrete distribution on $\rho$ [1]. On the other hand, one can define $H_{1}(x), H_{2}(x)$ and $H_{3}(x)$ by replacing $f(x)$ in Equations $(1.1)-(1.3)$ by $g_{j}(x ; \lambda)$. Hence we define entropy measures $H_{1}(x, \rho), H_{2}(x, \rho)$ and $H_{3}(x, \rho)$ of the partition $\rho$ after replacing $f(x)$ in Equations (1.1)-(1.3) by $\mu\left(S_{j} ; \lambda\right)$.

Definition 4. Let $H$ be an entropy measure and $f(x, y)=f_{1}(x) f_{2}(y)$ where $f(x, y)$ is the joint density of the random vector $(X, Y)$ then $H$ is said to be additive if

$$
H(f(x, y))=H\left(f_{1}(x)\right)+H\left(f_{2}(y)\right)[6]
$$

Sections 3 gives relation between each of $H_{i}, i=1,2,3$ entropy of partition sample for models and corresponding entropy of underlying models, also $\delta$-sufficient partition in the case of geometric distribution is introduced in such section. Sections 4 deals with the measure of the efficiency of a random sample with respected to a given entropy. In section 5 we derived analytical entropy for different discrete distributions. Section 6 gives simulation of data from different distribution and conducted efficiency of samples.

\section{THE MAIN RESULTS}

This section concerns with the main results in this paper. The results obtained are proving that the constructions of the mentioned entropy measures of information for models are comparable with that when the sample space of models is partitioned. Theorem (3.1) illustrated this idea.

Theorem 3.1. Let $X$ be a discrete random variable defined on the probability space $(\Omega, \mathcal{F}, P)$ and $\rho$ be F-partition of the space X,then

(a) $H_{1}(x) \geq H_{1}(x ; \rho)$ 
(b) $H_{2}(x) \geq H_{2}(x ; \rho)$

(c) $H_{3}(x) \geq H_{3}(x ; \rho)$

\section{Proof:}

(a)

$$
\begin{aligned}
H_{1}(x) & =E(f(x))^{\alpha-1}=\sum_{x} f(x)(f(x))^{\alpha-1} \\
& =\sum_{j=1}^{n} \sum_{x \in S_{j}} f(x)(f(x))^{\alpha-1} \\
& =\sum_{j=1}^{n}\left(\mu ( S _ { j } ) ( \mu ( S _ { j } ) ) ^ { \alpha - 1 } \sum _ { x \in S _ { j } } g _ { j } ( x ) \left(\left(g_{j}(x)\right)^{\alpha-1}\right.\right. \\
& =H_{1}(x ; \rho) H_{j}(x)
\end{aligned}
$$

Since $H_{j}(x) \geq 0$ for each $j$ [7] then (a) holds.

(b)

$$
\begin{aligned}
H_{2}(x) & =2^{\alpha E(\log f(x)} \text { for any } \alpha \geq 0, \alpha \neq 1 \\
& =2^{\alpha \sum_{x} f(x) \log f(x)} \\
& =2^{\alpha \sum_{j=1}^{n} \sum_{x \in S_{j}} \mu\left(S_{j}\right) g_{j}(x) \log \left(\mu S_{j}\right) g_{j}(x)} \\
& =2^{\alpha\left(\sum _ { j = 1 } ^ { n } \mu ( S _ { j } ) \operatorname { l o g } \left(\mu\left(S_{j}\right) \sum_{x \in S_{j}} g_{j}(x)+\sum_{j=1}^{n} \mu\left(S_{j}\right) \sum_{x \in S_{j}} g_{j}(x) \log \left(g_{j}(x)\right)\right.\right.} \\
& =H_{2}(x ; \rho)\left(2^{\alpha \sum_{x \in S_{j}} g_{j}(x) \log \left(g_{j}(x)\right.}\right)^{\sum_{j=1}^{n} \mu\left(S_{j}\right)} \\
& =H_{2}(x ; \rho)\left(H_{j}(x)\right)^{\sum_{j=1}^{n} \mu\left(S_{j}\right)}
\end{aligned}
$$

Since $H_{j}(x) \geq 0$ then $\left(H_{j}(x)\right)^{\sum_{j=1}^{n} \mu\left(S_{j}\right)} \geq 0$ therefore $(b)$ hold. 
(c)

$$
\begin{aligned}
H_{3}(x)= & \frac{1}{1-\alpha} \sum_{x} f(x)\left(f(x)^{\alpha-1}-1\right) \\
= & \frac{1}{1-\alpha}\left(\sum_{j=1}^{n} \mu\left(S_{j}\right) \sum_{x \in S_{j}} g_{j}(x)\left(\mu\left(S_{j}\right)^{\alpha-1} g_{j}(x)^{\alpha-1}-1\right)\right. \\
= & \frac{1}{1-\alpha}\left(\sum_{j=1}^{n} \mu\left(S_{j}\right)\left(\mu\left(S_{j}\right)\right)^{\alpha-1} \sum_{x \in S_{j}} g_{j}(x) g_{j}(x)^{\alpha-1}-1\right) \\
= & \frac{1}{1-\alpha}\left(\sum_{x \in S_{j}} g_{j}(x)\left(\mu\left(S_{j}\right)^{\alpha-1} g_{j}(x)^{\alpha-1}\right)\left(\sum_{j=1}^{n} \mu\left(S_{j}\right)\left(\mu\left(S_{j}\right)\right)^{\alpha-1}\right)-1\right) \\
& \left.+\sum_{x \in S_{j}} g_{j}(x)\left(g_{j}(x)\right)^{\alpha-1}-1\right) \\
= & \sum_{x \in S_{j}} g_{j}(x)\left(g_{j}(x)\right)^{\alpha-1} \frac{1}{1-\alpha}\left(\mu\left(S_{j}\right)\left(\mu\left(S_{j}\right)\right)^{\alpha-1}-1\right) \\
& +\frac{1}{1-\alpha}\left(\sum_{x \in S_{j}} g_{j}(x)\left(g_{j}(x)\right)^{\alpha-1}-1\right) \\
= & \sum_{x \in S_{j}} g_{j}(x)^{\alpha} H_{3}(x, \rho)+H_{j}(x)
\end{aligned}
$$

Since $g_{j}(x)^{\alpha} \geq g_{j}(x) \geq 0, \alpha>0, \alpha \neq 1$.

Therefore $\sum_{x \in S_{j}} g_{j}(x)^{\alpha} \geq \sum_{x \in S_{j}} g_{j}(x)=1$ and $H_{j}(x) \geq 0$ for each $j$.

Then (c) Hold.

Remark 3.1. In the case of modeling the reformative by continuous random variable, it is not clear that the results of Theorem (3.1) can be applied. Here we discuss only the case for exponential distribution. Suppose $X$ is random variable with p.d.f

$$
f(x)=\left\{\begin{array}{l}
\lambda e^{-\lambda x}, x>0, \lambda>0 \\
0, \text { otherwise }
\end{array}\right.
$$

Then $H_{i}(x)$ for $i=1,2,3$ can be derived as following:

$$
\begin{aligned}
H_{1}(x) & =\int_{0}^{\infty} \lambda e^{-\lambda x}\left(\lambda e^{-\lambda x}\right)^{\alpha-1} d x \\
& =\lambda^{\alpha} \int_{0}^{\infty} e^{-\alpha \lambda x} d x \\
& =\frac{\lambda^{\alpha-1}}{\alpha}
\end{aligned}
$$




$$
\begin{aligned}
H_{2}(x) & =2^{\alpha \int_{0}^{\infty} f(x) \log f(x) d x} \\
& =2^{\alpha \int_{0}^{\infty} \lambda e^{-\lambda x} \log \left(\lambda e^{-\lambda x}\right) d x} \\
& =2^{\alpha \int_{0}^{\infty} \lambda \log (\lambda) e^{-\lambda x} d x-\alpha \lambda^{2} \int_{0}^{\infty} x e^{-\lambda x} d x} \\
& =2^{\alpha(1-\log \lambda)} \\
H_{3}(x)= & \frac{1}{1-\alpha}\left(\int_{0}^{\infty} f(x)(f(x))^{\alpha-1} d x-1\right) \\
= & \left.\frac{1}{1-\alpha}\left(\int_{0}^{\infty} \lambda e^{-\lambda x}\left(\lambda e^{-\lambda x}\right)^{\alpha-1}\right) d x-1\right) \\
= & \frac{1}{1-\alpha}\left(\lambda^{\alpha}\left(\int_{0}^{\infty} e^{-\lambda \alpha x} d x-1\right)\right. \\
= & \frac{1}{1-\alpha}\left(\left(\frac{\lambda^{(\alpha-(\lambda / 2)}}{\alpha}\right)-1\right)
\end{aligned}
$$

Suppose the partition $\rho=\left[S_{1}=[0,1), S_{2}=[1, \infty)\right]$. Then

$$
\mu\left(S_{1}\right)=1-e^{-\lambda} \operatorname{and} \mu\left(S_{2}\right)=e^{-\lambda} .
$$

Hence it can be derived $H_{i}(x ; \rho)$ for $i=1,2,3$ as following:

$$
\begin{gathered}
H_{1}(x, \rho)=\left(1-e^{\lambda}\right)\left(1-e^{\lambda}\right)^{\alpha-1}+e^{-\lambda}\left(e^{-\lambda}\right)^{\alpha-1} \\
=\left(1-e^{-\lambda}\right)^{\alpha}+e^{-\alpha \lambda} \\
H_{2}(x ; \rho)=2^{\alpha \sum_{j=1}^{2} \mu\left(S_{j}\right) \log \mu\left(S_{j}\right)} \\
=2^{\alpha\left(\left(1-e^{-\lambda}\right) \log \left(1-e^{-\lambda}\right)+e^{-\lambda} \log e^{-\lambda}\right)} \\
=2^{\alpha\left(\left(1-e^{-\lambda}\right) \log \left(1-e^{-\lambda}\right)-\lambda e^{-\lambda}\right)} \\
\begin{aligned}
H_{3}(x, \rho)=\frac{1}{1-\alpha}\left(\sum_{j=1}^{2} \mu\left(S_{j}\right) \mu^{\alpha-1}\left(S_{j}\right)-1\right) \\
=\frac{1}{1-\alpha}\left(\left(1-e^{-\lambda}\right)\left(1-e^{-\lambda}\right)^{\alpha-1}+e^{-\lambda}\left(e^{-\lambda}\right)^{\alpha-1}-1\right) \\
=\frac{1}{1-\alpha}\left(\left(1-e^{-\lambda}\right)^{\alpha}+e^{-\alpha \lambda}-1\right)
\end{aligned}
\end{gathered}
$$

For example,take $\lambda=2.2$ and $\alpha=1.4$ then by using computer it can be shown that:

(a) $H_{1}(x)=0.9791, H_{1}(x ; \rho)=0.8944$. 
(b) $(b) H_{2}(x)=0.8144, H_{2}(x ; \rho)=0.4740$.

(c) $(c) H_{3}(x)=0.2378, H_{3}(x ; \rho)=0.2341$.

This means that Theorem (3.1) holds if $X$ is an exponential random variable. It is clear from the proof of Theorem (3.1) that if

$$
g_{j}(x)=\left\{\begin{array}{l}
1, x \in S_{j} \\
0, x \in S_{j}^{c}
\end{array}\right.
$$

i.e. $S_{j}$ is a singleton. This means that the partition consists of a singleton does not lose any amount of entropy. This condition is equivalent to the fact that an equality in all parts of the theorem(3.1) hold iff $H_{1}(x)=H_{2}(x)=H_{3}(x)=0$.

Example 1. ( $\delta$-Sufficient Partition)

Let $\mathrm{X}$ be random variable with p.d.f.

$$
f(x)=\left\{\begin{array}{l}
\lambda(1-\lambda)^{x-1}, x=1,2, \ldots \\
0, \text { otherwise }
\end{array}\right.
$$

Then

$$
\begin{aligned}
H_{1}(x) & =\sum_{x} \lambda(1-\lambda)^{x-1}\left(\lambda(1-\lambda)^{x-1}\right)^{\alpha-1} \\
& =\lambda^{\alpha} \sum_{x}(1-\lambda)^{\alpha(x-1)} \\
& =\frac{\lambda^{\alpha}}{1-(1-\lambda)^{\alpha}} \\
H_{2}(x) & =2^{\alpha \sum_{x} \lambda(1-\lambda)^{x-1}(\log (\lambda)+(x-1) \log (1-\lambda)} \\
& =2^{\alpha\left(\log (\lambda)+\log (1-\lambda)^{1 / \lambda}-\log (1-\lambda)\right.} \\
& =2^{\alpha \log \lambda(1-\lambda)^{1 / \lambda-1}}
\end{aligned}
$$

From Equation (3.7) then

$$
H_{3}(x)=(1 /(1-\alpha))\left(\left(\lambda^{\alpha} /\left(1-(1-\lambda)^{\alpha}\right)-1\right)\right.
$$

Assume the partition $\rho=S_{j}, j=1, \ldots, n$ such that

$$
S_{j}=\left\{\begin{array}{l}
(j), j=1,2, \ldots, n-1 \\
\mathcal{N}-(1,2, \ldots, n-1), j=n
\end{array}\right.
$$


where $\mathcal{N}$ is the set of natural numbers. Then

$$
\mu\left(S_{j}\right)=\left\{\begin{array}{l}
\lambda(1-\lambda)^{j-1}, j=1,2, \ldots, n-1 \\
(1-\lambda)^{n-1}, j=n
\end{array}\right.
$$

For this partition it can be shown that:

(i) $H_{1}(x ; \rho)=\left(1-(1-\lambda)^{n-1}\right) H_{1}(x)$.

(ii) $H_{2}(x ; \rho)=\left(1-(1-\lambda)^{\alpha(n-1)}\right) H_{2}(x)$.

The relative loss of entropy

(i) $H_{1}(x ; \rho) /\left(H_{1}(x)\right)=\left(1-(1-\lambda)^{n-1}\right)=1-\delta_{1}$ where $_{1}=(1-\lambda)^{n-1}$.

(ii) $H_{2}(x ; \rho) /\left(H_{2}(x)\right)=\left(1-(1-\lambda)^{\alpha(n-1)}\right)=1-\delta_{2}$ where $\delta_{2}=(1-\lambda)^{\alpha(n-1)}$.

Finally, the number of classes $\mathrm{n}$ which leads to a given $\delta_{1}$ and $\delta_{2}$ respectively is the smallest $\mathrm{n}$ such that:

(i) $n \geq 1+\left(\log \delta_{1}\right) / \log (1-\lambda)$ in case of the Vavrda and Charval measure.

(ii) $n \geq 1+\left(\log \delta_{2}\right) / \alpha \log (1-\lambda)$ in case of the Forte average certainty measure.

\section{EFFICIENCY OF A SAMPLE}

It is clear that entropy measures are additive [6]. This property will be used to measure the efficiency of a random sample of size n i.e. $S_{n}=\left\{X_{1}, \ldots, X_{n}\right\}$, where $X_{1}, \ldots, X_{n}$ are an independent and identically distributed (i.i.d) from the distribution $f(X ; \lambda)$. To see this let $S_{n}=\left\{X_{1}, \ldots, X_{n}\right\}$ where $X_{1}, \ldots, X_{n}$ are i.i.d, then the Forte entropy for a random sample $S_{n}$ is given by:

$$
H_{2}\left(S_{n}\right)=2^{\alpha E\left(\log f\left(X_{i} ; \lambda\right)\right.}
$$

Let $X$ be a random variable whose taking values in the set $C:=\left\{Y_{1}, \ldots, Y_{n}\right\}$. Assume that we have a random sample $S_{n}$ of size $n$, and let $\left\{x_{1}, x_{2}, \ldots, x_{k}\right\}$ be its values with frequencies $\left\{r_{1}, r_{2}, \ldots, r_{k}\right\}$, respectively, where $k \leq n$. Now the relative frequency of $x_{i}$ is:

$$
r\left(x_{i}\right)=r_{i} / n, i=1,2, \ldots, k
$$


Hence the estimated Forte entropy in the sample $S_{n}$ is denoted by:

$$
H_{2} E\left(S_{n}\right)=2^{\sum_{i=1}^{k} r\left(x_{i}\right) \log \left(r\left(x_{i}\right)\right.}
$$

The Forte entropy in $X$ is defined by Equation(4.1)and so the estimated relative efficiency in the sample $S_{n}$ is given by:

$$
\text { eff }=\left(H_{2} E\left(S_{n}\right)\right) /\left(H_{2}\left(S_{n}\right)\right)
$$

where eff is Forte relative efficiency.

It is known that the amount of entropy in a given sample size is measured by Equation (4.4) is less than or equal to $(1-\delta) 100$ where $\delta<1$

\section{MEASURES OF ENTROPY FROM DISTRIBUTIONS}

In this section entropy in Equation (1.2) can be derived for different distributions as following:

Example (5.1):

Case1: Geometric distribution

Let $Y: G(\lambda)$ with p.d.f

$$
f_{1}(y ; \lambda)=\left\{\begin{array}{l}
\lambda(1-\lambda)^{y-1}, y=1,2, \ldots \\
0, \text { otherwise }
\end{array}\right.
$$

To calculate Forte entropy, since

$\log \left(f_{1}(y ; \lambda)\right)=\log (\lambda)+(y-1) \log (1-\lambda)$,

hence,

$$
H_{2, G}(Y)=2^{\alpha E\left(\log \left(f_{1}(y ; \lambda)\right)\right)}=2^{(\alpha(\lambda \log (\lambda)+(1-\lambda) \log (1-\lambda)) / \lambda)}
$$

Let $Y_{1}, Y_{2}, \ldots, Y_{n}$ be a random sample from a geometric distribution with p.d.f given by Equation (5.1), let $S_{n}=\sum_{i=1}^{n} Y_{i}$, then Forte entropy for $S_{n}$ is given by:

$$
H_{2}\left(S_{n}\right)=n H_{2, G}(Y)
$$

Case 2: Binomial distribution

Let $Y_{1}, Y_{2}, \ldots, Y_{n}$ be a random sample from a Bernoulli distribution with p.d.f

$$
f(y ; \lambda)=\left\{\begin{array}{l}
\lambda^{y}(1-\lambda)^{n-y}, y \in 0,1 \\
0, \text { otherwise }
\end{array}\right.
$$


Let $X=\sum_{i=1}^{n} Y_{i}$; notice that $X: B(n, \lambda)$ with p.d.f

$$
f_{2}(x ; \lambda)=\left\{\begin{array}{l}
\lambda^{x}(1-\lambda)^{n-x}, x \in 0,1,2, \ldots n \\
0, \text { otherwise }
\end{array}\right.
$$

Then the Forte entropy is

$$
\begin{aligned}
H_{2, B}(X) & =2^{\alpha E\left(\log \left(f_{2}(x ; \lambda)\right)\right)} \\
& =2^{\alpha E(\log (x !(n-x) !))+\log (n !)+n \lambda \log (\lambda)+n(1-\lambda) \log (1-\lambda)}
\end{aligned}
$$

Case 3: Poisson distribution

Let $Y_{1}, Y_{2}, \ldots, Y_{n}$ be a random sample from a Poisson distribution with p.d.f

$$
f(y ; \lambda)=\left\{\begin{array}{l}
\epsilon^{-\lambda} \lambda^{y} / y !, y \in 0,1,2, \ldots \\
0, \text { otherwise }
\end{array}\right.
$$

Let $X=\sum_{i=1}^{n} Y_{i}$, that is for large $\mathrm{n}$ and small $\lambda, X \approx$ Poisson distribution with $n \lambda$ [3]. The approximate p.d.f of $X$ is given by:

$$
f_{3}(x ; \lambda)\left\{\begin{array}{l}
\epsilon^{-n \lambda}(n \lambda)^{x} / x !, x \in 0,1,2, \ldots \\
0, \text { otherwise }
\end{array}\right.
$$

From which

$\log \left(f_{3}(x ; \lambda)\right)=-n \lambda+x \log n \lambda-\log x !$,

Then the Forte entropy is:

$$
\left.H_{(} 2, P\right)(X)=2^{\alpha E\left(\log \left(f_{3}(x ; \lambda)\right)\right)}=2^{\alpha(n \lambda(\log (n \lambda)-1)-E(\log x !))}
$$

\section{COMPUTATIONS}

In this section the computations of the entropy measures for the different distributions that given analytically in Example (5.1) are computed by Matlab 8.1 program. The estimate of the entropy in Equation (1.2) and the efficiency in the sample $S_{n}$ are given by Equations (4.4) in each Case 1,2 and 3.

(a) To illustrate the concepts, as in Example (5.1) Case 1, 80 random samples are simulated from geometric distributions $G(\lambda)$. For each $\lambda=0.1: 0.1: 0.8$ and for each $n=10: 10: 100$, in each case the efficiency eff of each simulated sample is computed in Table1 from Equation (5.2) and Equation(4.4). 
Table 1: Estimated efficiency of sample of size $n$ from geometric distribution with parameter $\lambda$ by Forte entropy

\begin{tabular}{|c|c|c|c|c|c|c|c|c|}
\hline$\lambda$ & 0.1 & 0.2 & 0.3 & 0.4 & 0.5 & 0.6 & 0.7 & 0.8 \\
\hline 10 & 0.15446 & 0.22543 & 0.21405 & 0.35191 & 0.28166 & 0.34027 & 0.39301 & 0.60933 \\
\hline 20 & 0.13064 & 0.10777 & 0.17507 & 0.31483 & 0.28315 & 0.29369 & 0.38382 & 0.57309 \\
\hline 30 & 0.07339 & 0.11759 & 0.18120 & 0.24226 & 0.32979 & 0.32979 & 0.36189 & 0.58942 \\
\hline 40 & 0.07048 & 0.08037 & 0.16107 & 0.17983 & 0.27625 & 0.31383 & 0.45408 & 0.61546 \\
\hline 50 & 0.06544 & 0.09632 & 0.16708 & 0.22753 & 0.27189 & 0.43636 & 0.39484 & 0.55483 \\
\hline 60 & 0.05558 & 0.09061 & 0.14213 & 0.23119 & 0.23010 & 0.31167 & 0.44196 & 0.57877 \\
\hline 70 & 0.06359 & 0.08790 & 0.16111 & 0.18684 & 0.28986 & 0.31616 & 0.53940 & 0.54976 \\
\hline 80 & 0.05462 & 0.10354 & 0.14410 & 0.19057 & 0.30088 & 0.31724 & 0.43167 & 0.50541 \\
\hline 90 & 0.04491 & 0.09053 & 0.13819 & 0.22812 & 0.29907 & 0.45302 & 0.44199 & 0.54872 \\
\hline 100 & 0.05389 & 0.08396 & 0.13052 & 0.20579 & 0.29462 & 0.41369 & 0.40726 & 0.54991 \\
\hline
\end{tabular}

(b) For estimating the efficiency, as in Example (5.1) Case 2 also 80 random samples are simulated from binomial distributions $X: B(n, \lambda)$. For each $\lambda=0.1: 0.1: 0.8$ and for each $n=10: 10: 100$. In each case the efficiency eff of each simulated sample is computed in Table 2 from the Equation(5.4) and Equation (4.4).

Table 2: Estimated efficiency of sample of size $\mathrm{n}$ from Binomial distribution with parameter $\lambda$ by Forte entropy.

\begin{tabular}{|c|c|c|c|c|c|c|c|c|}
\hline$\lambda$ & 0.1 & 0.2 & 0.3 & 0.4 & 0.5 & 0.6 & 0.7 & 0.8 \\
\hline 10 & 0.06933 & 0.05822 & 0.04923 & 0.04920 & 0.06605 & 0.05135 & 0.05614 & 0.06385 \\
\hline 20 & 0.06167 & 0.05091 & 0.04785 & 0.04303 & 0.04631 & 0.04689 & 0.04721 & 0.04477 \\
\hline 30 & 0.05116 & 0.04352 & 0.03884 & 0.04201 & 0.03653 & 0.04051 & 0.03968 & 0.04352 \\
\hline 40 & 0.04638 & 0.04360 & 0.03885 & 0.04005 & 0.03949 & 0.04211 & 0.04278 & 0.04313 \\
\hline 50 & 0.05193 & 0.04051 & 0.03894 & 0.03786 & 0.03776 & 0.03833 & 0.04153 & 0.04253 \\
\hline 60 & 0.05055 & 0.04190 & 0.03805 & 0.03660 & 0.03670 & 0.03562 & 0.03727 & 0.04101 \\
\hline 70 & 0.04913 & 0.04034 & 0.03415 & 0.03439 & 0.03579 & 0.03823 & 0.03639 & 0.04171 \\
\hline 80 & 0.04932 & 0.04175 & 0.03697 & 0.03483 & 0.03733 & 0.03770 & 0.03691 & 0.03980 \\
\hline 90 & 0.04852 & 0.03949 & 0.03417 & 0.03398 & 0.03811 & 0.03707 & 0.03789 & 0.03886 \\
\hline 100 & 0.04816 & 0.03987 & 0.03806 & 0.03416 & 0.03447 & 0.03498 & 0.03883 & 0.04226 \\
\hline
\end{tabular}

(c) The Poisson distribution $X: P(\lambda)$ is used for simulated 70 random samples as in Example (5.1) Case 3, for each $\lambda=1: 0.5: 4$ and for each $n=5: 3: 32$. In each case the efficiency eff of each simulated sample is computed in Table 3 from the Equation (5.5) and Equation(4.4) 
Table 3: Estimated efficiency of sample of size $n$ from Poisson distribution with parameter $\lambda$ by Forte entropy.

\begin{tabular}{|c|c|c|c|c|c|c|c|}
\hline$\lambda$ & 1 & 1.5 & 2.0 & 2.5 & 3.0 & 3.5 & 4.0 \\
\hline 5 & 0.12593 & 0.26548 & 0.40503 & 0.23914 & 0.26548 & 0.40503 & 0.33525 \\
\hline 8 & 0.16649 & 0.39248 & 0.27234 & 0.37602 & 0.31595 & 0.43609 & 0.33241 \\
\hline 11 & 0.17339 & 0.29248 & 0.33775 & 0.29406 & 0.38921 & 0.38144 & 0.47658 \\
\hline 14 & 0.31559 & 0.37608 & 0.39613 & 0.38673 & 0.40554 & 0.46148 & 0.45538 \\
\hline 17 & 0.31014 & 0.37717 & 0.46299 & 0.42698 & 0.41523 & 0.38594 & 0.42195 \\
\hline 20 & 0.21923 & 0.36355 & 0.39295 & 0.46163 & 0.46040 & 0.47590 & 0.50738 \\
\hline 23 & 0.23126 & 0.35479 & 0.39660 & 0.44763 & 0.44908 & 0.41081 & 0.49610 \\
\hline 26 & 0.32007 & 0.36868 & 0.47891 & 0.40604 & 0.44984 & 0.47940 & 0.50051 \\
\hline 29 & 0.31776 & 0.36143 & 0.44049 & 0.43275 & 0.43869 & 0.45561 & 0.50973 \\
\hline 32 & 0.29083 & 0.33681 & 0.40185 & 0.46179 & 0.41407 & 0.41140 & 0.50051 \\
\hline
\end{tabular}

\section{CONCLUSIONS}

The calculations of the estimated relative efficiency of entropy that given in [6] are derived for the cases where the distribution of sample size that selected are geometric, binomial and Poisson. These calculations that given by Tables 1 to 3 in Example (5.1)show that in all these three cases from 1 to 3 the relative efficiency lying at range 1 , i.e from 0 to 1 as: $0 \leq$ eff $<1$. Moreover, in Table1, Case1 the geometric distribution gives that when $\lambda<0.5$ :

(i) eff $<0.3600$ for all values of $\mathrm{n}$.

(ii) In any fixed value of $\mathrm{n}$, eff is increasing when $\lambda$ is increasing.

In Case 2 the binomial distribution when $\lambda=0.1: 0.1: 0.8$ Table 2 gives that:

(i) eff is not exceeding 0.06933 .

(ii) eff almost is decreasing when $\mathrm{n}$ is increasing at any value of $\lambda$.

Case 3 of Poisson distribution with $\lambda=1: 0.5: 4$, Table 3 gives that:

(i) eff is not exceeding 0.50973 .

(ii) The efficiency in range less than 0.4 , i.e $0.12593 ;$ eff $; 0.50973$ then the sample size for this efficiency in range $5 \leq$ ef $f \leq 32$. 


\section{ACKNOWLEDGMENT}

The author would like to thank Professor I. H. Elsanousi for his comments, suggestions and recommendations.

\section{REFERENCES}

[1] A. M. Awad, On the Fisher $\beta$-sufficient partition, Communication in Statistics(A), Theory and Methods ,Vol.27(9),(1992),2645-2664.

[2] A. M. Awad, Entropy of a partition and efficiency of a sample, Abhath AlYarmouk pure sci. and eng., Vol. 5, (1996), pp. 9-30.

[3] M. M. Azzam , A. M. Awad, Entropy measures and some distribution approximations, Microelectron Reliab., Vol. 36,No 10,(1996),1569-1580.

[4] M. Behara, and J.Chawla, Generalized Gamma-entropy. In Selecta. Statistician Canadian, Vol.2, (1974),15-38.

[5] B. Forte, Measures of information in general axiomatic theory, R.I.R.O.Serie R-3, Vol.2, (1969),63-90.

[6] M. E. Gobar, Efficiency of a random sample from some distributions, Global journal of mathematics, Vol.6, No 3, (2016), 667-673.

[7] C. E. Shannon and W. Weaver, The mathematical theory of communication, Univ. III. Press, Urbana Chicago, (1949).

[8] J. Vavrda, and F. Charval, Quantification method of classification processes: Concept of structural ?-entropy, Kybernetika, Vol.1, 3, (1967), 30-35. 